\title{
Investigation of the effect of adjuvant chemotherapy in stage II colorectal cancers
}

\author{
Hormoz MAHMOUDVAND, Abdolhassan TALAEIZADEH, Hojatallah SHAHBAZIAN, Khatereh ANBARI, Sareh \\ JAHANBAKHSH, Sedigheh NADRI
}

\begin{abstract}
Colorectal cancer is the most prevalent and malignant gastrointestinal cancer and the fourth most common cancer around the world. Selection of the treatment method is based on the staging of the disease. The aim of this interventional study is to investigate the effect of adjuvant chemotherapy on local recurrence, distant metastases, and three-year disease-free lifespan in patients with stage II colorectal cancer.

This study conducted between 2001 and 2007 involved 40 patients with stage II colorectal cancer. Subsequent to surgery, the adjuvant chemotherapy was performed employing the FOLFOX4 diet therapy; the patients were further examined every 3 months using tumor marker counts, sonography, CT scan and colonoscopy for identification of any local recurrence
\end{abstract}

and metastasis. Finally, at the end of the third year and after chemotherapy, the rates of local recurrence, metastasis and survival of the patients were investigated.

The present study was conducted on 40 patients, 23 of whom were male and the rest were female. After three years of follow-up, 2 patients had local recurrence and 7 patients had distant metastasis. All of the patients survived throughout the three-year follow-up period.

In this study, the adjuvant chemotherapy in stage II colorectal cancer led to significant overall survival and three-year diseasefree survival.

Keywords: Adjuvant chemotherapy; stage II colorectal cancer; overall survival.
Hormoz Mahmoudvand

1. Razi Herbal Medicines Research Center, Lorestan University of Medical Sciences, Khorramabad, Iran

2. Department of Surgery, Lorestan University of Medical Sciences, Khorramabad, Iran

Abdolhassan Talaeizadeh

Department of Cancer Surgery, Jundishapour University of Medical Sciences, Ahvaz, Iran

Hojatallah Shahbazian

Department of Clinical Oncology, Jundishapour University of Medical Sciences, Ahvaz, Iran

Khatereh Anbari

Social Determinant of Health Research Center, Faculty of Medicine, Lorestan University of Medical Sciences, Khorramabad, Iran.

Sareh Jahanbakhsh

Research Center for Tropical and Infectious Diseases Research Cente, Kerman University of Medical Sciences, Kerman, Iran.

Sedigheh Nadri

Department of Anesthesiology, Lorestan University of Medical Sciences, Khorramabad, Iran

Corresponding Author:

Sedigheh Nadri

e-mail:dr.s.nadri@gmail.com

Submitted / Gönderilme: 14.03.2017 Revised / Düzeltme: 07.06.2017 Accepted / Kabul: $\quad 08.06 .2017$

How to cite this article : Mahmoudvand $\mathrm{H}$, Talaeizadeh A, Shahbazian H, Anbari K, Jahanbakhsh S, Nadri S. Investigation of the effect of adjuvant chemotherapyinstageIIcolorectalcancers.MarmaraPharmJ2017;21(4):998-1001

\section{Introduction}

Colorectal cancers are the most common gastrointestinal malignancy [1] and the fourth most common cancer all around the world [2]. The National Comprehensive Cancer Network (NCCN) has declared that in 2009, almost 106100 new cases of colon cancer and about 40870 cases of rectal cancer have occurred in the USA; further, in the same year, nearly 49920 cases of death caused by these cancers have been recorded [3]. There are no similar statistics for Iran. Regardless of the present statistics, the mortality rate caused by the colorectal cancers has decreased over the past three decades, which was probably due to faster diagnosis, proper screening and use of better treatment methods [3]. With regard to the nonspecific nature of most of the clinical symptoms such as abdominal pain, bowel habits changes, melena, hematochezia, unjustifiable fatigue and anemia, reducing the morbidity and mortality rates is currently achieved using screening methods such as colonoscopy, double-contrast barium enema, sigmoidoscopy, fecal occult blood testing and virtual colonoscopy, which is the best and golden standard colonoscopy [4]. Choosing the treatment method is based on staging of the disease and surgery is the 
base of the definitive treatment in the early stages. Today, chemotherapy treatment and radiotherapy in some cases (such as rectal cancers) are used as neoadjuvant therapies as well as adjuvant treatments in certain stages of the disease. Different studies have been conducted in the cancer surgery center regarding the type of diet therapy and medicines as well as their consumption times (adjuvant or neoadjuvant) [5]. One of the controversial cases of the adjuvant therapies is in the second stage of the colorectal cancers. American Cancer Society suggests one of these three options: monitoring the patients especially those with low risk, performing the adjuvant chemotherapy especially in patients with high risk, performing the clinical trial on new treatment protocols $[6,7]$. The aim of this interventional study is to investigate the effect of adjuvant chemotherapy on the rate of local recurrence, distant metastasis and three-year diseasefree survival in patients with stage II colorectal cancer.

\section{Materials and Methods}

\section{Patient and study design}

This research was conducted on all the patients with stage II colorectal cancer undergoing surgery and adjuvant chemotherapy during 2001-2009 from General Hospitals of Lorestan Province, Western Iran. Patients who were in stage II before and after the surgery based on pathological staging were enrolled. Information regarding gender, cancer location (right colon and cecum, left colon, total colon, recto sigmoid), incidence of distant metastasis were collected in a predesigned questionnaire. Then, the adjuvant chemotherapy was preformed employing FOLFOX4 diet therapy for six months (as fortnightly for 12 sessions each session two days). The drug used are FU-5 ( 400 and $\left.600 \mathrm{mg} / \mathrm{m}^{2}\right)$, leucovorin $\left(200 \mathrm{mg} / \mathrm{m}^{2}\right)$, and oxaliplatin $\left(85 \mathrm{mg} / \mathrm{m}^{2}\right)$; besides, the patients were examined every 3 months using tumor marker counts, sonography and CT scan (every 6 months), as well as colonoscopy (the first year after surgery and then three years later) for identification of any local recurrence and metastasis. Finally, at the end of the third year after chemotherapy, the local recurrence, metastasis and survival of the patients were investigated. By examining the effect of adjuvant chemotherapy in stage II colorectal cancer, the necessary information were extracted from the patient records and inserted into a questionnaire designed for this purpose.

This study was approved by Ethics Committee of Lorestan University of Medical Sciences (Permit No. 90/236). In addition, a written informed consent was obtained from all the participants before surgery. The written consent of the patients was obtained after providing full explanations on the research, possible complications and methods of treating the complications. The patients were allowed to leave the study in case of dissatisfaction and discontentment to continue their cooperation in the project at any stage of the research. The patients' information was kept completely confidential and there was a complete preparation for treatment by the research group in case of incidence of any complication.

\section{Statistical analysis}

Analytical and descriptive statistics was carried out using SPSS 17.0 software (SPSS Inc., Chicago, IL, USA). Descriptive statistics were reported in terms of per cent (for categorical) and mean (SD) (for continuous) variables. Chi-square test was applied to access the association between independent variables and outcome. $\mathrm{P}<0.05$ was considered to be statistically significant.

\section{Results}

The mean \pm SD age of the patients was $52.97 \pm 12.64$ years. Out of 40 patients, 23 were male and 17 were female. At three year follow-up time, only two (5\%) patients were found to have local recurrence, while 38 patients had none. The frequency of local recurrence did not differ between both genders and it was similar for all cancer locations ( $p>0.05$ for both). After three-year follow-up period, 33 (17.5\%) patients were found to have no distant metastases, while 7 had distant metastasis. All of the patients survived through the three-year follow-up period. The findings regarding the incidence of metastasis according to the location of the cancer are shown in Table 1.

Table-1. Frequency of distant metastasis based on metastasis cancer location

\begin{tabular}{cc}
\hline Cancer location & $\begin{array}{c}\text { Presence of distant metastasis; } \\
\text { No. }(\%)\end{array}$ \\
\hline Right colon and cecum $(\mathrm{n}=8)$ & $2(25)$ \\
Left colon $(\mathrm{n}=5)$ & $1(20)$ \\
Total colon $(\mathrm{n}=3)$ & $1(33.33)$ \\
Rectum $(\mathrm{n}=15)$ & $3(20)$ \\
Sigmoid $(\mathrm{n}=9)$ & $0(0)$ \\
Total $(\mathrm{n}=40)$ & $7(17.5)$ \\
\hline
\end{tabular}

Cancer location did not have an effect on the rate of distant metastasis $(\mathrm{p}>0.05)$. 


\section{Discussion}

In the present research, only the patients with stage II colorectal cancer undergoing the adjuvant chemotherapy have been investigated. Age and gender were found to have no effect on the outcomes of adjuvant chemotherapy at stage II colorectal cancer in terms of distant metastasis or local recurrence. However, other studies on the colorectal cancers revealed that age had an impact on the outcomes of therapy [8].

Due to the low number of the subjects, the above-mentioned findings cannot absolutely yield the conclusion that whether the age and gender could affect the local recurrence, distant metastasis, and the survival or not. In a study by Hermosillo et al., old patients qualified for receiving the adjuvant chemotherapy in colorectal cancers didn't experience significant changes in their living conditions [9]. The outcomes of chemotherapy regarding the local recurrence and distant metastasis were similar for cancers of different locations. The tumor's location seems to have no major effect on the patients' prognosis; nevertheless, achieving more accurate judgments in this regard requires a larger sample size as well as a phase II clinical trial. Although the statistical tests on the relationship of distant metastasis and local recurrence have shown no significant statistical difference, it seems that the patients without distant metastases had no local recurrence as well; however, due to the low number of the samples, it is impossible to make a definite judgment in this regard and a study with larger sample size is required. In this study, the three-year survival of the subjects was $100 \%$; although, in the literature different survival rates ranging from 55 to $80 \%$ have been mentioned for stage II [10]. For instance, on average, in Korea, USA, and Australia, the lifespan has been 5 years [11-13], respectively in 75\%, 87\% and $87 \%$ of the cases. The average follow-up period was 5 years in these studies; while, it was three-years in the present study. In the study by Johnson, the five-year survival rate of the stage II patients after a surgery without chemotherapy was $70-80 \%$ [14].

By performing four clinical trials, the NSABBP Foundation showed the relative advantages of the adjuvant chemotherapy in increasing the overall lifespan and the disease-free survival in both stages II and III of the colorectal cancers [15]. These findings were consistent with the meta-analysis performed in Mayo Clinic on 3341 patients with and without metastasis to lymph nodes [16]. The Japanese Cancer Association showed the positive impact of the oral fluoropyrimidine on improvement of the overall lifespan and disease-free survival in each of the three stages of colorectal cancer [17]. In a report on the study by Gary et al. 3238 stage II patients from 150 medical centers in 17 countries around the world, FU-5 therapy was found to have positive impact on reducing the local recurrence by $1-5 \%$ and the improvement of the overall lifespan and disease-free survival [18]. By investigating the available resources for the adjuvant therapy in the stage II patients, The American Society of Clinical Oncology showed that, despite the improvement of the disease-free survival by the adjuvant therapy, there was no considerable improvement in the overall lifespan of these patients; thus, the researchers suggested that the adjuvant therapies should be performed on the patients who have been selected properly [19]. In the studies entitled MOSAIC on the clinical trial of comparing the injective FU-5 adjuvant medicine diet with the FU$5+$ Oxiplatine (FOLFOX4) medicine diet, it was shown that three-year disease-free survival in 1100 patients undergoing FOLFOX has been improved significantly.

In a study on the adjuvant treatment of stage II and III colorectal cancer, Makata et al. declared that DST-CD was useful for predicting the response of the FU-5-based adjuvant chemotherapy treatment in the patients with stages II and III colorectal cancers [20]. The notable point is that this research was conducted on about 40 subjects; furthermore, since there was no group without adjuvant therapy in this study, it was impossible to make a definitive opinion on the effect of the adjuvant chemotherapy on incidence of distant metastases, local recurrence and five-year disease-free survival.

In general, results of the present study were obtained from a phase II clinical trial and in this regard, other similar studies with larger sample size and meta-analysis with cases without chemotherapy are required. However, it should be mentioned the limitations of the present study are small sample size, short follow-up period, and lack of controls.

\section{Acknowledgments}

The authors would like to extend their sincere appreciation to all of the colleagues and the clinical research unit of Shohadaye Ashayer Hospital, particularly to Ms. Elham Sheikhi who assisted them in this project.

\section{Conflict of interest}

The authors declare that there is no conflict of interest regarding the publication of this paper 


\section{References}

1. McCleary NJ, Benson AB, Dienstmann R. Personalizing Adjuvant Therapy for Stage II/III Colorectal Cancer. Am Soc Clin Oncol Educ Book 2017; 37: 232-45.

2. Wilkinson N. Surgical therapy for colorectal adenocarcinoma. Gastroenterol Clin North 2008; 37: 253-67.

3. Kuan FC, Lai CH, Ku HY, Wu CF, Hsieh MC, Liu TW, Yeh CY, Lee KD. The survival impact of delayed surgery and adjuvant chemotherapy on stage II/III rectal cancer with pathological complete response after neoadjuvant chemoradiation. Int J Cancer 2017; 140: 1662-9.

4. Xu Z, Mohile SG, Tejani MA, Becerra AZ, Probst CP, Aquina CT, Hensley BJ, Arsalanizadeh R, Noyes K, Monson JR, Fleming FJ. Poor compliance with adjuvant chemotherapy use associated with poorer survival in patients with rectal cancer: An NCDB analysis. Cancer 2017; 123:52-61.

5. Andre T, Boni C, Mounedji-Boudiaf L, Navarro M, Tabernero J, Hickish T, Topham C, Zaninelli M, Clingan P, Bridgewater J, Tabah-Fisch I, de Gramont A; Multicenter International Study of Oxaliplatin/5-Fluorouracil/Leucovorin in the Adjuvant Treatment of Colon Cancer (MOSAIC) Investigators. Oxaliplatin, fluorouracil, and leucovorin as adjuvant treatment for colon cancer. N Engl J Med 2004; 23: 2343-51.

6. Zwierzina H. Adjuvant therapy of stage II colorectal cancer who will benefit from treatment? Memo-magazine of Eur Med Oncol 2008; 1:75-7.

7. Edge S, Byrd DR, Compton CC, Fritz AG, Greene FL, Trotti A. AJCC Cancer Staging Manual 2011; 45-81. New York.

8. Lieffers JR, Baracos VE, Winget M, Fassbender K. A comparison of Charlson and Elixhauser comorbidity measures to predict colorectal survival using administrative health data. Cancer 2011; 117: 1957-65.

9. Hermosillo-Rodriguez JL, Anaya DA, Sada Y, Walder A, Amspoker AB, Berger DH, Naik AD. The effect of age and comorbidity on patient-centered health outcomes in patients receiving adjuvant chemotherapy for colon cancer. Eur J Cancer 2003; 39:2214-22.

10. McLeish JAL, Thursfield VJ, Giles GG. Survival from colorectal cancer in Victoria: 10-Year follow-up of the 1987 management survey. ANZ J Surg 2002; 72:352-6.

11. Gatta G, Ciccolallo L, Capocaccia R, Coleman MP, Hakulinen T, Møller H, Berrino F; EUROCARE Working Group. Differences in colorectal cancer survival between Europe and U.S. populations: The importance of sub-site and morphology. Eur J Cancer 2003; 39: 2214-22.
12. Bittorf B, Merkel S, Merkel U, Matzel KE, Wein A, Dimmler A, Hohenberger W. Primary Signet-Ring cell carcinoma of the colorectum. Langenbeck's Arch Sur 2004; 389:178-83

13. Mahmoudvand H, Forutani S, Nadri S. Comparison of Treatment Outcomes of Surgical Repair in Inguinal Hernia with Classic versus Preperitoneal Methods on Reduction of Postoperative Complications. Biomed Res Int 2017;2017:3785302.

14. Park YJL, Park KJ, Park JG, Lee KU, Choe KJ, Kim JP. Prognostic factors in 2230 Korean colorectal cancer patients: Analysis of consecutively operated cases. World J Surg 1999; 23:721- 6 .

15. Johnston PG. Stage II Colorectal Cancer: To Treat or not to Treat. Oncologist 2005; 10:332-4.

16. Mamounas EL, Wieand S, Wolmark N, Bear HD, Atkins JN, Song K, Jones J, Rockette H. Comparative efficacy of adjuvant chemotherapy in patients with Dukes' B versus Dukes' C colon cancer: results from four National Surgical Adjuvant Breast and Bowel Project adjuvant studies (C-01, C-02, C-03, C-04). J Clin Oncol 1999; 17:1349-55.

17. Gill SL, Loprinzi CL, Sargent DJ, Thomé SD, Alberts SR, Haller DG, Benedetti J, Francini G, Shepherd LE, Francois Seitz J, Labianca R, Chen W, Cha SS, Heldebrant MP, Goldberg RM.. Pooled analysis of fluorouracil-based adjuvant therapy for stage II and III colon cancer; who benefits and by how much? J Clin Oncol 2004; 22: 1797-1806.

18. Sakamoto JL, Hamada C, Kodaira S, Nakazato H, Ohashi Y. Adjuvant therapy with oral fluoropyrimidines after curative resection for colorectal cancer: Individual patient data metaanalysis of randomized trials. Jpn J Clin Oncol 1999; 29:78-86.

19. Gray RG, Barnwell J, Hills R, McConkey C, Williams N, Kerr D. QUASAR: a randomized study of chemotherapy (CT) vs observation including 3238 colorectal cancer patients. Proc Am Soc Clin Oncol 2004; 22: 3501-6.

20. Benson AB, Schrag D, Somerfield MR. American Society of Clinical Oncology recommendations on adjuvant chemotherapy for Stage II cancer. J Clin Oncol 2004; 22: 340819.

21. Mekata E, Sonoda H , Shimizu T, Tatsuta T, Yamaguchi T, Endo Y, Tani T. Clinical predictive value of in vitro anticancer drug sensitivity test for the therapeutic effect of adjuvant chemotherapy in patients with stage II-III colorectal cancer. Mol Clin Oncol 2013; 1: 763-7. 